\title{
DELETED PRODUCTS OF CONES
}

ARTHUR H. COPELAND, JR. ${ }^{1}$

1. Introduction. A difference between homotopy theory and isotopy theory, in some ways the truly basic difference, is that homotopy theory does not distinguish between the contractible spaces, while isotopy theory does. Thus the contractible spaces stand out as a promising starting point for a study of isotopies. Among the contractible spaces, the cones $\hat{X}$ over finitely triangulable spaces seem to be the most amenable to investigation. Moreover, additional knowledge of these cones is exactly what is needed to further the study of isology functors [1].

An important tool used in isotopy theory is the deleted product functor or residual functor. This is the functor $R$ whose value on a topological space $X$ is $R X=X \times X$-diagonal; it is extended over the category of embeddings in the obvious way. This functor has been studied by $\mathrm{Hu}[3],[4]$, Patty [6], [7] and [8], Shapiro [9] and Wu $[10]$. The main theorem of the present paper relates the cohomology groups of $X, R X$ and $R \hat{X}$ when $X$ is a finitely triangulable space. Roughly speaking, the relation is

$$
H^{m}(R X)=H^{m}(X)+H^{m}(X)+H^{m+1}(R \hat{X})
$$

(direct sum). This result holds in all dimensions for most spaces, and in most dimensions for all spaces. The difficult dimension is the top one. The recalcitrant spaces include the spheres and certain other manifolds. For orientable differentiable manifolds the matter may be settled by examining the Thom class of the tangent bundle.

The cohomology theory used throughout this paper will be reduced singular theory with integer coefficients. Since this is the only theory used, the customary tilde over the $H$ has been omitted.

2. Cohomology decompositions. If $A$ and $B$ are subsets of a space $X$ and if $i_{A}: A \rightarrow X$ and $i_{B}: B \rightarrow X$ are the inclusion maps, let

$$
\alpha=\alpha(A, B ; m): H^{m}(X) \rightarrow H^{m}(A)+H^{m}(B)
$$

be the homomorphism given by the formula

$$
\alpha(u)=\left(i_{A}^{*}(u), i_{B}^{*}(u)\right), \quad u \in B^{m}(X) .
$$

Received by the editors December 12, 1963.

1 This research was supported in part by the Army Research Office (Durham), contract number DA-ARO(D)-31-124-G147. 
A space is called $m$-decomposable if it has proper subsets $A, B \subset X$ such that $\alpha(A, B ; m)$ has a left inverse. Note that if $X$ is not connected, then it is $m$-decomposable for all $m$.

THEOREM 2.1. If $X$ is a finitely triangulable n-dimensional space, then $X$ is $m$-decomposable for each $m \neq n$.

Proof. The space is trivially $m$-decomposable for $m>n$. Let $A$ be an $n$-simplex of $X$ and let $B$ be the complement of the interior of $A$. Then $A \cap B$ is an $(n-1)$-sphere, and the two end groups in the following segment of the Mayer-Vietoris sequence are trival for $m<n-1$.

$$
H^{m-1}(A \cap B) \rightarrow H^{m}(X) \stackrel{\alpha(A, B ; m)}{\longrightarrow} H^{m}(A)+H^{m}(B) \rightarrow B^{m}(A \cap B) .
$$

Thus the conclusion holds in this range. When $m=n-1, H^{m-1}(A \cap B)$ $=0$ and $H^{m}(A \cap B)$ is projective, and once more $\alpha(A, B ; m)$ has a left inverse.

In order for a finitely triangulable $n$-dimensional space $X$ to fail to be $n$-decomposable, each point of $X$ must lie in an $n$-simplex. Otherwise, taking $A$ to be the union of the (closed) $n$-simplexes and $B$ to be the empty set would cause $\alpha(A, B ; n)$ to be an isomorphism. Furthermore, any two $n$-simplexes $\sigma$ and $\tau$ of $X$ can be joined by a string $\sigma_{0}=\sigma, \sigma_{1}, \cdots, \sigma_{k}=\tau$ of $n$-simplexes such that $\sigma_{i} \cap \sigma_{i-1}$ is an $(n-1)$-simplex for $i=1,2, \cdots, k$, for otherwise, let $A$ be one stringcomponent of $X$ and let $B$ be the closure of $X-A$. Since $A \cap B$ lies in the $(n-2)$-skeleton of $X$, the Mayer-Vietoris sequence of $(X ; A, B)$ shows $\alpha(A, B ; n)$ to be an isomorphism. These remarks show that $X$ must be a Cantor manifold, for if an $(n-2)$-dimensional subset separated $X$, it would have to separate some set of the form $\sigma_{0} \cup \sigma_{1} \cup \ldots$ $\cup \sigma_{k}$ where $\sigma_{0}, \cdots, \sigma_{k}$ is a string of $n$-simplexes, and this is impossible.

On the other hand, some Cantor manifolds are $n$-decomposable. An $n$-cell is $n$-decomposable because its cohomology vanishes; the figure eight provides an example with nontrivial cohomology in dimension $n(n=1)$.

No compact, connected $n$-manifold is $n$-decomposable, for it has nontrivial $n$-dimensional cohomology, while each proper subset has trivial cohomology in dimension $n$.

The examples presented so far have $X$ n-decomposable exactly when $H^{n}(X)$ fails to be a cyclic group. The following example dispels a possible conjecture. Let $X$ be a space obtained by attaching two $n$-cells $E$ and $E^{\prime}$ to an $(n-1)$-sphere $S$ by means of maps of degrees $p$ and $p^{2}$, respectively ( $p$ any positive integer). Then $H^{n}(X)$ 
$=Z+Z_{p}$ (the direct sum of an infinite cyclic group and one of order $p$ ). Let $A$ be a proper subset of $X$ and let $x \in X-A$. The inclusion map $i_{A}$ may be factored

$$
B^{n}(X) \rightarrow H^{n}(X-\{x\}) \rightarrow H^{n}(A) .
$$

We have the following values for $H^{n}(X-\{x\})$, depending upon where $x$ is located:

$$
H^{n}(X-\{x\})= \begin{cases}H^{n}\left(E^{\prime} \cup S\right)=Z_{p^{2}} & \text { if } x \in E, \\ H^{n}(E \cup S)=Z_{p} & \text { if } x \in E^{\prime} \\ 0 & \text { if } x \in S .\end{cases}
$$

Thus if $A$ and $B$ are proper subsets, then the image of $\alpha(A, B ; n)$ is finite, and $\alpha(A, B ; n)$ can not have a left inverse.

A notion dual to $m$-decomposability can be defined using homology. The dual to Theorem 2.1 then holds only for $m \neq n, n-1$. An $n$ dimensional triangulable space with two independent $n$-cycles is $n$ decomposable in homology.

\section{Cohomology of deleted products of cones.}

THEOREM 3.1. If $X$ is a finitely triangulable $n$-dimensional space, then $H^{m}(R X)$ is isomorphic to the direct sum

$$
H^{m}(X)+H^{m}(X)+H^{m+1}(R \hat{X})
$$

for $m \neq n, n-1$. If, in addition, $X$ is $n$-decomposable, then this relation holds for $m=n$ and $n-1$.

The result is stated in terms of singular theory, but since $X$ is finitely triangulable, $R X$ and $R \hat{X}$ have the homotopy types of finitely triangulable spaces, whence the same relation holds for any reduced integral cohomology theory (in the sense of Eilenberg-Steenrod [2]). The proof of Theorem 3.1 is preceded by two lemmas.

Let $X$ be given a triangulation which will be held fixed throughout this section. Let $J X \subset X \times X$ be the space

$$
J X=\bigcup\{\sigma \times \tau: \sigma, \tau \text { are simplexes of } X, \sigma \cap \tau=\varnothing\} .
$$

The inclusion map $J X \rightarrow R X$ is a homotopy equivalence [3]. Let $I=[0,1]$ be the unit interval, let $\dot{I}=\{0,1\}$ be its boundary, and let

$$
\phi: X \times X \times I \rightarrow X * X
$$

be a quotient map defining the join $X * X$ of $X$ with itself. We choose $\phi$ to be one-to-one on $X \times X \times(0,1)$ and such that 


$$
\begin{aligned}
& \phi\left(x_{1}, x_{2}, 0\right)=\phi\left(x_{1}, x_{2}^{\prime}, 0\right), \quad \text { and } \\
& \phi\left(x_{1}, x_{2}, 1\right)=\phi\left(x_{1}^{\prime}, x_{2}, 1\right)
\end{aligned}
$$

for $x_{1}, x_{1}^{\prime}, x_{2}, x_{2}^{\prime} \in X$.

LEMma 3.2. The space $J \hat{X}$ is homeomorphic with $\phi[J X \times I \cup X \times X \times \dot{I}]$ $\subset X * X$.

Proof. Let $\theta: X \times I \rightarrow X$ be the quotient map sending $X \times\{1\}$ into the apex $v$ of the cone $\hat{X}$, and let $M=\phi[J X \times I \cup X \times X \times \dot{I}]$. Note that

$$
\begin{aligned}
J X= & X \times\{v\} \cup\{v\} \times X \\
& \cup \cup\{\theta(\sigma \times I) \times \tau \cup \sigma \times \theta(\tau \times I): \sigma \cap \tau=\varnothing\}
\end{aligned}
$$

when we identify $X$ with $\theta[X \times\{0\}]$. If $\sigma$ and $\tau$ are disjoint simplexes of $X$, let $f_{\sigma \tau}: \sigma \times \tau \times I \rightarrow J X$ be the map defined by

$$
f_{\sigma \tau}\left(x, x^{\prime}, t\right)= \begin{cases}\left(x, \theta\left(x^{\prime}, 1-2 t\right)\right), & 0 \leqq t \leqq \frac{1}{2}, \\ \left(\theta(x, 2 t-1), x^{\prime}\right), & \frac{1}{2} \leqq t \leqq 1,\end{cases}
$$

when $x \in \sigma, x^{\prime} \in \tau, t \in I$. Observe that $f_{\sigma \tau}$ is an embedding. It follows from the definition of $J X$ that the maps $f_{\sigma \tau}$ fit together to form a map

$$
f: M \rightarrow J X \text {. }
$$

One routinely verifies that $f$ is a homeomorphism.

The subsets $X_{1}=\phi[X \times X \times\{0\}]$ and $X_{2}=\phi[X \times X \times\{1\}]$ are homeomorphic to $X$. Homeomorphisms $g_{i}: X_{i} \rightarrow X$ are given by the formulas

$$
\begin{aligned}
& g_{1}\left(\phi\left(x, x^{\prime}, 0\right)\right)=x, \\
& g_{2}\left(\phi\left(x, x^{\prime}, 1\right)\right)=x^{\prime},
\end{aligned}
$$

for $x, x^{\prime} \in X$. Let $p_{1}, p_{2}: R X \rightarrow X$ be the restrictions to $R X$ of the projection maps $X \times X \rightarrow X$. The symbol $i$ will be used to denote inclusion maps; the context will indicate which one. The direct sum group $H^{m}(X)+H^{m}(X)$ is abbreviated to $H^{m}(X)^{2}$.

LEMмA 3.3. There exist homomorphisms $\Delta$ such that the sequence

$$
\ldots \rightarrow H^{m}(R X) \stackrel{\beta}{\rightarrow} H^{m}(X)^{2} \stackrel{\alpha}{\rightarrow} H^{m}(R X) \stackrel{\Delta}{\rightarrow} H^{m+1}(R X) \rightarrow \cdots
$$

is exact when

$$
\begin{aligned}
\beta(u) & =\left(\left(g_{1}^{*}\right)^{-1} i_{u}^{*},\left(g_{2}^{*}\right)^{-1} i u\right), \quad u \in H^{m}(R X), \\
\alpha(v, w) & =p_{1}^{*}(v)-p_{2}^{*}(w), \quad v, w \in H^{m}(X) .
\end{aligned}
$$

Proof. Let 


$$
\begin{aligned}
C_{1} & =\phi\left[J X \times\left[0, \frac{1}{2}\right] \cup X \times X \times\{0\}\right], \\
C_{2} & =\phi\left[J X \times\left[\frac{1}{2}, 1\right] \cup X \times X \times\{1\}\right], \text { and } \\
N & =\phi\left[J X \times\left\{\frac{1}{2}\right\}\right] .
\end{aligned}
$$

Let $h_{i}: C_{i} \rightarrow X$ be maps defined by

$$
\begin{aligned}
& h_{1}\left(\phi\left(x, x^{\prime}, t\right)\right)=x, \\
& h_{2}\left(\phi\left(x, x^{\prime}, t\right)\right)=x^{\prime},
\end{aligned}
$$

for $x, x^{\prime} \in X, t \in I$. Let $g\left(\phi\left(x, x^{\prime}, \frac{1}{2}\right)\right)=\left(x, x^{\prime}\right) \in R X$ for $\left(x, x^{\prime}, \frac{1}{2}\right) \in N$. Note that $h_{1}, h_{2}$ are homotopy equivalences and that $g$ is a homeomorphism of $N$ onto a deformation retract of $R X$. The top row of the diagram below is the Mayer-Vietoris sequence of the triad $\left(M ; C_{1}, C_{2}\right)$.

$$
\begin{aligned}
& \cdots \rightarrow B^{m}(M) \stackrel{\beta^{\prime}}{\longrightarrow} H^{m}\left(C_{1}\right)+B^{m}\left(C_{2}\right) \stackrel{\alpha^{\prime}}{\rightarrow} H^{m}(N) \stackrel{\Delta^{\prime}}{\rightarrow} H^{m+1}(M) \rightarrow \cdots \\
& \uparrow f^{*} i^{*} \quad \uparrow h_{1}^{*}+h_{2}^{*} \quad \uparrow g^{*} \quad \uparrow f^{*} i^{*} \\
& \cdots \rightarrow H^{m}(R \hat{X}) \underset{\beta}{\longrightarrow} H^{m}(X)^{2} \underset{\alpha}{\longrightarrow} H^{m}(R X) \underset{\Delta}{\longrightarrow} H^{m+1}(R \hat{X}) \rightarrow \cdots
\end{aligned}
$$

The diagram is commutative, and the vertical homomorphisms are all isomorphisms.

Proof OF TheOREM 3.1. The result follows at once from Lemma 3.3 when $m>n$. Suppose $X$ is $m$-decomposable, and let $A, B \subset X$ be proper subsets such that $\alpha(A, B ; m)$ has a left inverse,

$$
\gamma_{0}: H^{m}(A)+H^{m}(B) \rightarrow H^{m}(X) .
$$

Let $a \in X-A$ and $b \in X-B$ be points, and set

$$
Y=(A \times\{a\}) \cup(\{a\} \times A) \cup(B \times\{b\}) \cup(\{b\} \times B) \subset R X .
$$

Suppressing the inclusion maps, we may write

$$
H^{m}(Y)=B^{m}(A \times\{a\})+H^{m}(\{a\} \times A)+H^{m}(B \times\{b\})+H^{m}(\{b\} \times B) .
$$

If $u \in H^{m}(R X)$ and if $i: Y \rightarrow R X$ is the inclusion map, let

$$
i^{*} u=v_{1} \times a+a \times v_{2}+w_{1} \times b+b \times w_{2},
$$

and set

$$
\gamma u=\left(\gamma_{0}\left(v_{1}, w_{1}\right),-\gamma_{0}\left(v_{2}, w_{2}\right)\right) \in H^{m}(X)^{2} .
$$

Then $\gamma \alpha$ is the identity on $H^{m}(X)^{2}$.

Thus if $X$ is $m$ - and $(m+1)$-decomposable, the sequence of Lemma 3.3 splits, and we have

$$
H^{m}(R X)=H^{m}(X)^{2}+H^{m+1}(R \hat{X}) .
$$


From Theorem 2.1, we see that this happens for all $m<n-1$. If $X$ is $n$-decomposable, the splitting occurs for all $m$.

Note that $H^{n-1}(X)^{2}$ always appears as a direct summand in $H^{n-1}(R X)$.

4. Cones over manifolds. If $X$ is an oriented differentiable $n$-manifold, then $(X \times X, R X)=\left(X^{2}, R X\right)$ may be regarded as the Thom complex of the tangent space of $X$. If $u \in H^{n}\left(X^{2}, R X\right)$ is the Thom class, then

$$
\phi(x)=p_{1}^{*}(x) \cup u=p_{2}^{*}(x) \cup u, \quad x \in H^{m}(X),
$$

defines isomorphisms

$$
\phi: H^{m}(X) \rightarrow H^{m+n}\left(X^{2}, R X\right)
$$

for all $m>0$ (if an ordinary cohomology theory is used, rather than a reduced one, then $\phi$ is an isomorphism for all $m)$. If $v \in H^{n}(X)$ is the appropriate generator and if $i: X^{2} \rightarrow\left(X^{2}, R X\right)$ is the inclusion map, then

$$
i^{*} u=1 \times v+v \times 1+w \in H^{n}\left(X^{2}\right),
$$

where $w$ lies in the image of

$$
\sum\left\{H^{p}(X) \otimes H^{q}(X): 0<p<n, p+q=n\right\}
$$

under the Künneth isomorphism. The class $w=w(X)$ is uniquely determined by the topological type of $X$.

TheOREM 4.1. If $X$ is an orientable differentiable $n$-manifold, then $w(X) \neq 0$ if and only if

$$
H^{m}(R X)=H^{m}(X)^{2}+H^{m+1}(R \hat{X})
$$

for $m=n, n-1$.

Proof. It suffices to show that

$$
\alpha: H^{n}(X)^{2} \rightarrow H^{n}(R X)
$$

has a left inverse, or, equivalently, that $\alpha$ is monic and $\operatorname{im} \alpha$ is a direct summand. If $(x, y) \in H^{n}(X)^{2}$, let $\bar{\alpha}(x, y)=x \times 1-1 \times y \in H^{n}\left(X^{2}\right)$. If $j: R X \rightarrow X^{2}$ is the inclusion map, then $\alpha=j^{*} \bar{\alpha}$. Note that $\bar{\alpha}$ is monic and im $\bar{\alpha}$ is a direct summand in $H^{n}\left(X^{2}\right)$. But ker $j^{*}$ is the cyclic group generated by $i^{*} u=v \times 1+1 \times v+w(X)$. Thus $\left(\operatorname{ker} j^{*}\right)$ $\bigcap(\operatorname{im} \bar{\alpha})=0$ if and only if $w(X) \neq 0$.

A similar result may be obtained for cohomology mod 2 when $X$ is a nonorientable manifold. The cohomology class $w(X)$ has been 
studied for the case of cohomology with coefficients in a field, $F$. It vanishes if and only if all cup products,

$$
H^{p}(X ; F) \otimes H^{n-p}(X ; F) \rightarrow H^{n}(X ; F),
$$

are trivial for $p=1,2, \cdots, n-1[5]$.

\section{BIBLIOGRAPHY} 216.

1. A. H. Copeland, Jr., Isology theory, Trans. Amer. Math. Soc. 107 (1963), 197-

2. S. Eilenberg and N. E. Steenrod, Foundations of algebraic topology, Princeton Univ. Press, Princeton, N. J., 1952.

3. S.-T. Hu, Isotopy invariants of topological spaces, Proc. Roy. Soc. Ser. A. 255 (1960), 331-366.

4. - Homotopy and isotopy properties of topological spaces, Canad. J. Math. 13 (1961), 167-176.

5. J. Milnor, Lectures on characteristic classes (mimeographed), Princeton Univ. Press, Princeton, N. J., 1957.

6. C. W. Patty, Homotopy groups of certain deleted product spaces, Proc. Amer. Math. Soc. 12 (1961), 369-373.

7. - The homology of deleted products of trees, Duke Math. J. 29 (1962), $413-428$.

8. - The fundamental group of certain deleted product spaces, Trans. Amer. Math. Soc. 105 (1962), 314-321.

9. A. Shapiro, Obstructions to the imbedding of a complex in a euclidean space. I. The first obstruction, Ann. of Math. (2) 66 (1957), 256-269.

10. W.-T. Wu, On the realization of complexes in euclidean spaces. I, Acta. Math. Sinica 5 (1955), 505-552.

NORTHWESTERN UNIVERSITY 